\title{
The Power of confocal Raman-AFM and Raman-SEM (RISE) Imaging in Polymer Research
}

\author{
Ute Schmidt ${ }^{1}$, Wei Liu ${ }^{2}$, Jianyong Yang ${ }^{2}$, T. Dieing ${ }^{1}$, and Klaus Weishaupt ${ }^{1}$ \\ 1. WITec GmbH, Ulm, Germany \\ 2. WITec Instruments Corp., Knoxville, TN, USA
}

Polymers play an essential role in modern materials science. Due to the wide variety of mechanical and chemical properties of polymers, they are used in almost every field of application and are still a dynamic area in the development of new materials. For many of these developments knowledge about the morphology and chemical composition of heterogeneous polymeric materials on a sub-micrometer scale is crucial. In the past two decades, AFM (atomic force microscopy) was one of the main techniques used to characterize the morphology and phase separations in thin polymeric films. This measuring technique however relies only on mechanical contrast, leaving research on similar polymers out of reach. The combination of a confocal Raman microscope with an AFM one decade ago provided the ability to unequivocally determine the chemical composition of a material. By acquiring Raman spectra at every image pixel, the high spatial and topographic resolution obtained with an AFM can be directly linked to the chemical information provided by confocal Raman spectroscopy [1,2]. In polymer science, Raman spectra provide quantitative information about various features such as: chemical nature (structural units, type and degree of branching, end groups, additives), conformational order (physical arrangement of the polymer chain), state of the order (crystalline, mesomorphous, and amorphous phases), and orientation (type and degree of polymer chain and side group alignment in anisotropic materials). A milestone in microscopy, achieved last year, is the combination of SEM (scanning electron microscopy) with confocal Raman imaging. For polymer research an SEM provides the morphology and the crystalline structure of polymer composites together with the atomic composition mainly carbon and hydrogen. The combination with confocal Raman imaging allows the allocation of the unique chemical composition of the high resolution structures with diffraction limited resolution.

The aims of this presentation are on the one hand to present results obtained with a confocal RamanAFM and a confocal Raman-SEM on the other hand, and highlight the benefits of such combined measuring capabilities. With the confocal Raman-AFM a three component polymer blend consisting of PS:EHA:SBR in the blending ratio 1:1:1 was analyzed. Figure 1 shows the high resolution topographic (a) and viscoelastic properties (b). The structures visible however are not present in the ratio of the blending. The Raman image (c) and the corresponding spectra (d) reveal an overlay of the EHA and SBR phase. Further information regarding substrate wetting and phase formation which could be derived by combining the results of both techniques will be presented. With the confocal Raman-SEM a polymer blend of PS:PMMA (blending ration 2:1) spin coated on a glass substrate was analyzed. Fig. 2 shows an overlay of a color coded Raman image consisting of PS:PMMA with the SEM image acquired from the same sample area. The SEM image reveals the fine structure of the two polymeric phases, whereas the chemical identification of the polymeric phases is provided by the confocal Raman image.

\section{References:}

[1] U. Schmidt, S. Hild, W. Ibach, O. Hollricher, Macromol. Symp. (2005), 230, p. 133

[2] U. Schmidt, J. Müller, J. Koenen, in "Confocal Raman Microscopy", ed. T. Dieing, O. Hollricher and J. Toporski, (Springer Series in Optical Sciences 158, Berlin-Heidelberg 2010) p.237. 

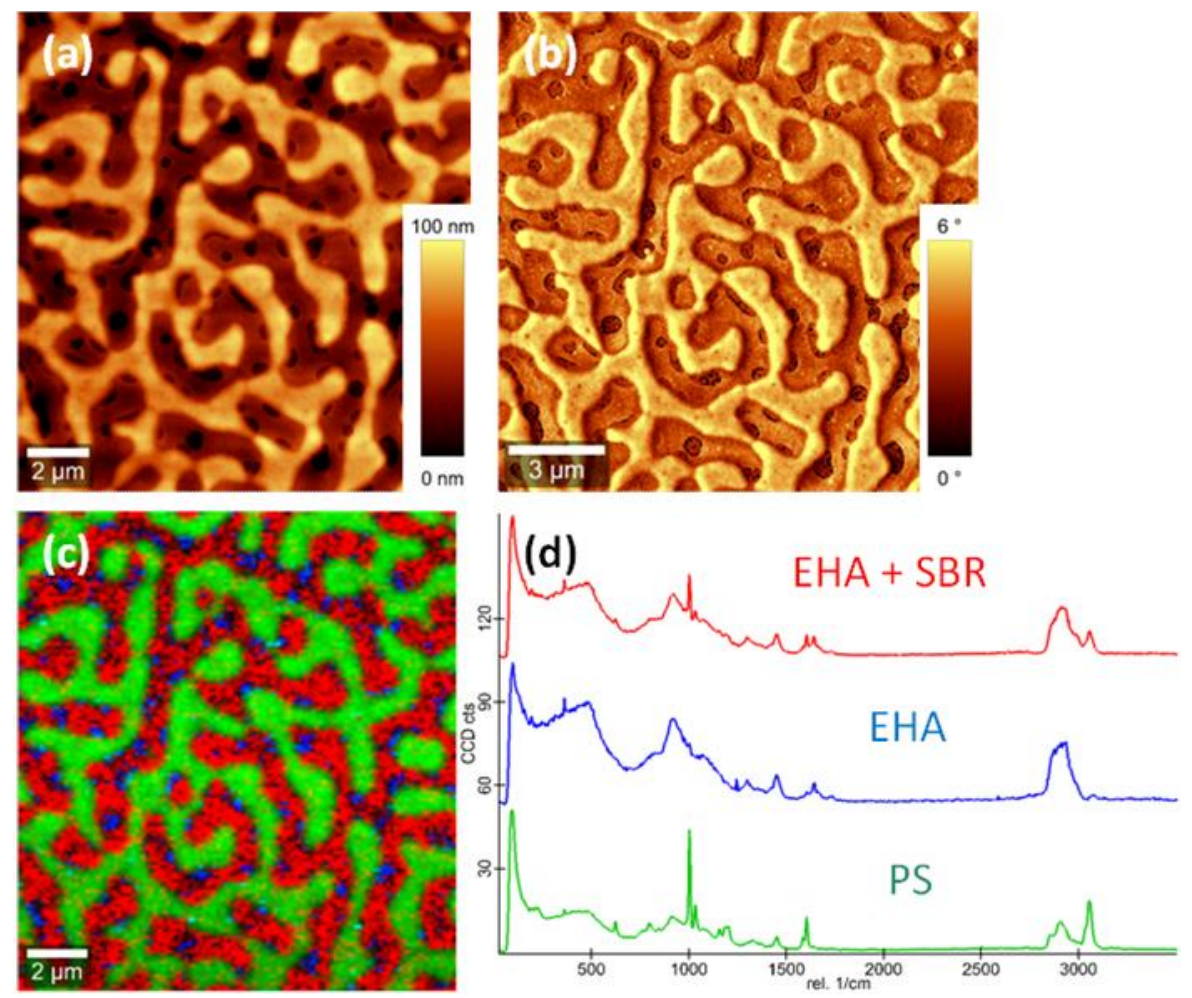

Figure 1. Confocal Raman-AFM images of a three component polymer blend PS:EHA:SBR (1:1:1): AFM topography (a), AFM phase (b), color coded Raman image (c) and corresponding spectra (d).
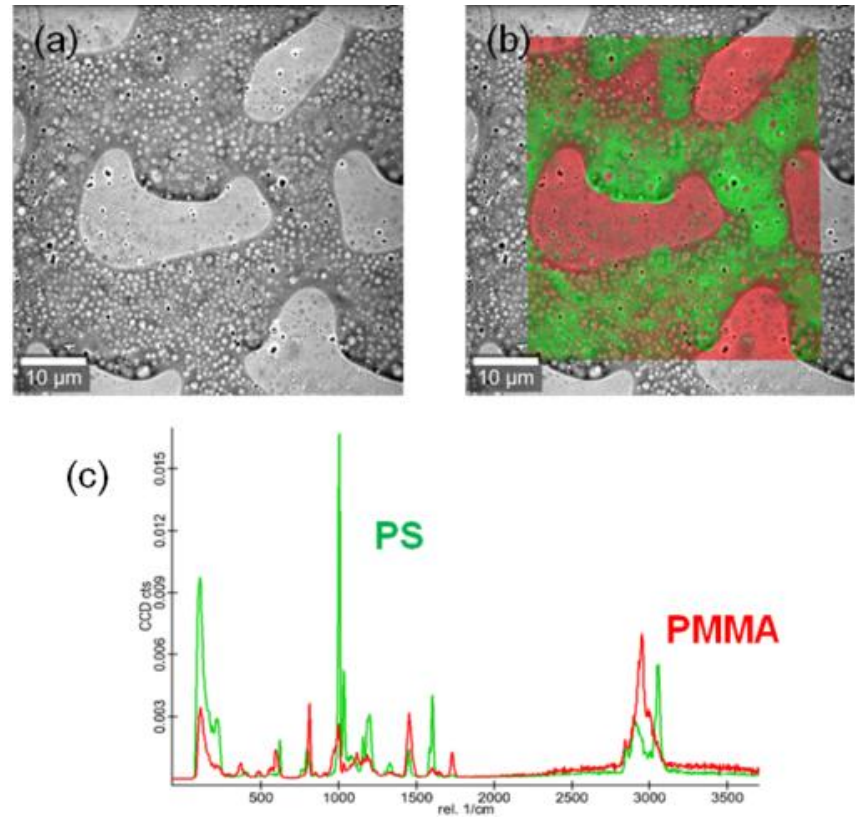

Figure 2. Confocal Raman-SEM imaging of a two component polymer blend PS:PMMA: SEM image (a), color coded Raman image overlaid on the SEM image (b), and corresponding spectra (c). 